\title{
RESPONSES IN LIGHT, SOUND AND SCENT*
}

\section{A Therapeutic Interactive Yoga System}

\author{
Sidney Fels \\ Department of Electrical and Computer Engineering \\ University of British Columbia \\ ssfels@ece.ubc.ca
}

James Gauthier

Department of Computer Science

University of British Columbia

gauthier@cs.ubc.ca

\author{
Patricia Smith \\ Faculty of Education \\ University of British Columbia \\ patkay@interchange.ubc.ca
}

\begin{abstract}
We describe an interactive system that uses gesture recognition to enhance the yoga experience through visual, auditory and olfactory feedback. Ancient theories associated with Kundalini yoga provide the theoretical basis for this research. The sensory feedback provided by the Therapeutic Interactive Yoga System promotes an immersive, multimedia experience that corresponds to the system of sensory stimuli outlined by chakra theory. As a user performs yoga, the system monitors the user's body and responds with colour, sound and scent appropriate to the user's yoga postures. Testing reveals that the Yoga System succeeds in producing an "enjoyable" and "relaxing" environment — one that seems to enhance the personal, meditative and therapeutic experience of yoga.
\end{abstract}

Keywords: Interactive systems, sensory feedback, olfactory feedback, yoga, yogic postures, energy centres, chakras, experiential interface.

*We would like to thank ATR MI\&C Research Laboratories Japan for their support of the Human Communication Technologies (HCT) research laboratory at the University of British Columbia, along with Ashbury's Aromatherapy, Richmond, BC, Canada for providing Spa Scenter aromatherapy equipment.

The original version of this chapter was revised: The copyright line was incorrect. This has been corrected. The Erratum to this chapter is available at DOI: 10.1007/978-0-387-35660-0_65 


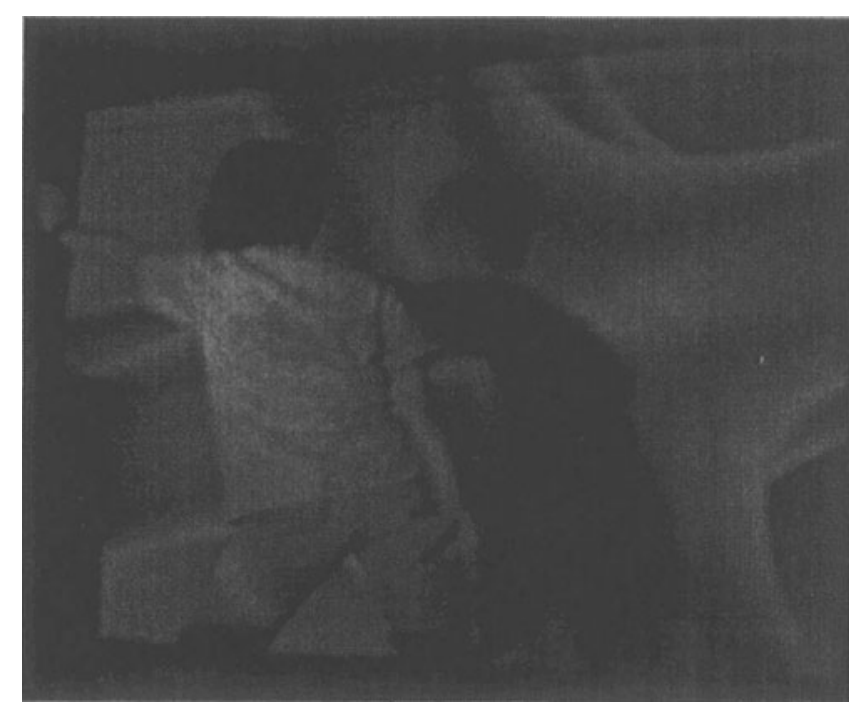

Figure 1. The yoga system being used. A user demonstrates the interactive nature of the system by performing a yogic posture and observing the corresponding projected video image.

\section{Introduction}

According to the tradition of Kundalini yoga, the human body possesses seven main energy centres, also known as chakras [10]. Each chakra is the intersection of energetic pathways within the body, and specific organs and mental states are associated with each chakra. The consistent practice of yoga seeks to open the chakras and improve energy circulation throughout the body. Kundalini theory also postulates that the body's chakras correspond to certain visual, auditory and olfactory stimuli - namely, the seven pitches of the C Major scale, the seven colour groups of the visual spectrum, and seven basic aromatherapy scents. These concepts form the theoretical basis of the Therapeutic Interactive Yoga System.

The research objective was to develop an interactive system capable of heightening the yogic experience so that it might enhance the therapeutic qualities of yoga. We combined aspects of yogic theory, the concept of "human energy centres" and various Human-Computer Interaction design techniques in our system. Our system monitors user's movements and provides the user with the appropriate visual, auditory and olfactory feedback in order to deepen the meditative experience according to the theory. If one accepts that the regular practice of yoga benefits the human body because it assists in systematically activating the body's chakras, then the Therapeutic Interactive Yoga System seeks to enhance the yoga experience by further stimulating these energy centres. 


\begin{tabular}{lllll} 
Position & Chakras & Colours & Pitch & Scent \\
\hline 1. & Crown & Violet & B & Lavender \\
2. & Third Eye & Indigo & A & Patchouli \\
3. & Throat & Blue & G & Geranium \\
4. & Heart & Green & F & Eucalyptus \\
5. & Navel & Yellow & E & Rosemary \\
6. & Spleen & Orange & D & Melissa \\
7. & Root & Red & C & Ylang-ylang (No scent used)
\end{tabular}

Table 1. The relationship between chakra, colour, pitch and scent. "Crown" refers to the top of the head. "Third eye" refers to the middle of the forehead. We piloted 4 yoga postures/asanas $(1,3,5$, and 7$)$ and their associated feedback as indicated by the highlighted entries.

\section{Yoga, Gesture Recognition and Sensory Feedback}

Several special qualities inherent in the practice of yoga make it particularly well-suited for an interactive multimedia system. Yoga consists of precise postures that happen slowly with long hold times. This makes yoga a good candidate for a gesture recognition system and an excellent domain for testing sensory feedback that evolves slowly — in particular, olfactory and musical feedback.

Because yoga happens slowly, the mood surrounding the yoga participant can "evolve" at a pace that allows auditory and olfactory displays to be effective. Also, yoga postures are well-defined and the gestural language is already learned. Combined with the slow time scale that defines the transition from one posture to another, this greatly simplifies gesture recognition. Thus, on both the input side (recognition of postures) and the output side (sensory feedback), yoga is a good match with a multi-sensory, interactive system.

Yoga, a Sanskrit word that literally means "union" or "joining together," has as its purpose the unification of body, emotion and mind by activating the chakras. Chakras can be interpreted as energy centres. Kundalini theory claims that each chakra may be activated or stimulated by a particular yoga posture or set of postures [1]. Four such postures are shown in figure 2. Also associated with each chakra is a colour, a pitch and a scent [6]. Some physiologists claim that each chakra holds the energy key to its associated area of the body [12].

The correspondence between chakras, yoga gestures, colours, sounds and scents is shown in table 2. Accordingly, our system associates multisensory feedback with each yoga gesture that it recognizes. For our system we chose four yoga gestures to recognize. (There are seven in the theory, but we did our pilot study with only four.) These have four associated sounds, colours and scents. By tracking the user's body position, the four gestures are recognized and the feedback appropriate to the theory is presented. The feedback is intended to be ambient so that the user's attention is not drawn to the changes 


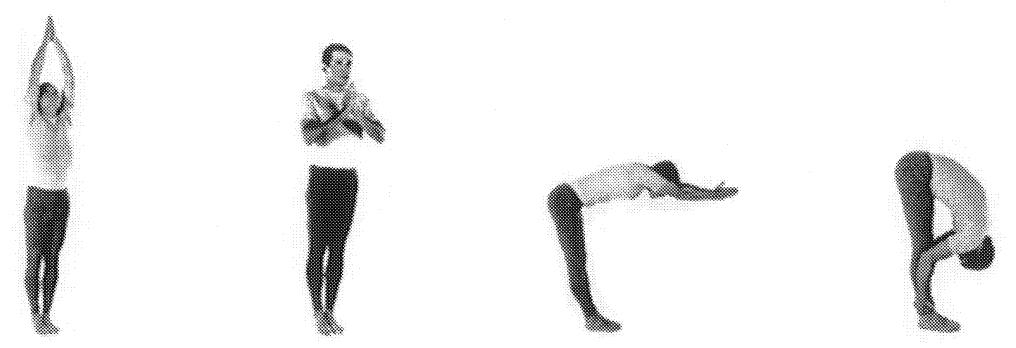

Figure 2. The four yoga positions (asanas) used during testing. These correspond to the Crown, Throat, Navel and Root chakras shown in table 2. The images are from [7].

in the environment. This is done to be consistent with the idea that yoga is a meditative, relaxing experiences and the environment around the user should not be distracting.

\section{Related Work}

Our yoga system attempts to use appropriate feedback in a whole body, interactive multimedia system to enhance the yoga experience or be a therapeutic device. Other systems have had related goals and suggest that this direction bodes well for the future of interactive multimedia. For example, the BioMuse integrates measured biopotentials to produce intimate sounds [2]. In Tosa's Interactive Poem [11] participants work with MUSE, a computer agent, to explore and create a poetic experience. The interaction and multimedia display helps to make the experience more intimate and deep. The Interactive Virtual Aerobic Trainer [4] provides an interactive system for enhancing the exercise experience. This system exploits the relatively stylized gestures in the exercise domain to make recognition easier just as in our system. Ultimately, its goal is to promote health. Virtual reality systems have been used for therapy as well, specifically, for the treatment of arachnophobia [3]. The Iamascope [5] provides an interactive multimedia experience that creates a pleasing, calming experience. the use of colour, mandala-like imagery, and sound matched to movement makes it work well. While not interactive, video-based mandalas have been used for relaxation [13][9]. Of course, video-based systems are popular to assist the yoga practitioner [8]. Our system fits well within the user-centered view that successful interactive multimedia requires attention to multiple senses.

\section{System Design}

Figure 3 shows a block diagram of our system. For input, we use three magnetic trackers (Fastrak, Polhemus Inc.). Data from these sensors are fed to the gesture recognition (GR) module for recognition described below. Once 


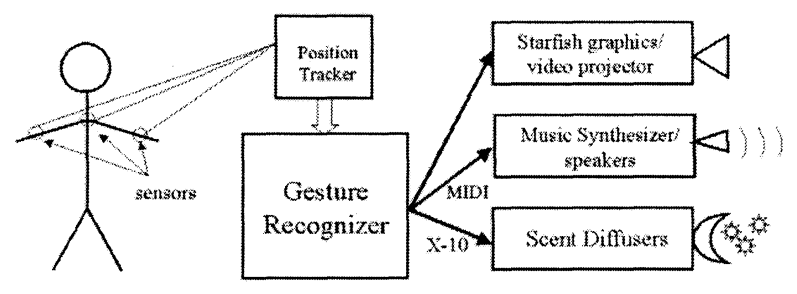

Figure 3. Block diagram of the Yoga system. The gesture recognition (GR) module process reads the data from the Fastrak sensors and communicates with three display processes. The GR process is also responsible for the training procedure.

recognized, the appropriate feedback is displayed as shown in table 2 . We have three different output modalities, video, audio and olfactory. The GR module and the interface software run on a single Linux computer with a $500 \mathrm{MHz}$ Pentium2 CPU.

\subsection{Gesture Recognition}

It was possible to make our gesture recognition system very simple because of the very specific, well-learned gestures in yoga (see figure 2) as well as the slow movements in yoga. Thus, we use only Euclidean distance as our metric to compare each new received set of of sensor values to our gesture training set. Each sensor reading yields three positions of the sensors attached to the arms and body of the user as shown in figure 3. Each position consists of a measurement of the $\mathrm{X}, \mathrm{Y}$ and $\mathrm{Z}$ coordinates of the sensors. Thus, for each measurement we have a vector of nine numbers.

Prior to using the system in its normal mode, users are put through a simple, quick training routine. The training routine prompts the user to make each pose once. While they are holding a pose, the position of their body is recorded by the GR module. The training set is a vector of the three positions measured for each gesture. We use only one sample value of each position for our training set. The four examples in the training set are used for the recognition as described above.

The gesture recognition proceeds as follows: first, read a new set of sensor values. Second, calculate the distance of the new sample between each of the four samples in the training set. Whichever is closest is the recognized gesture. 


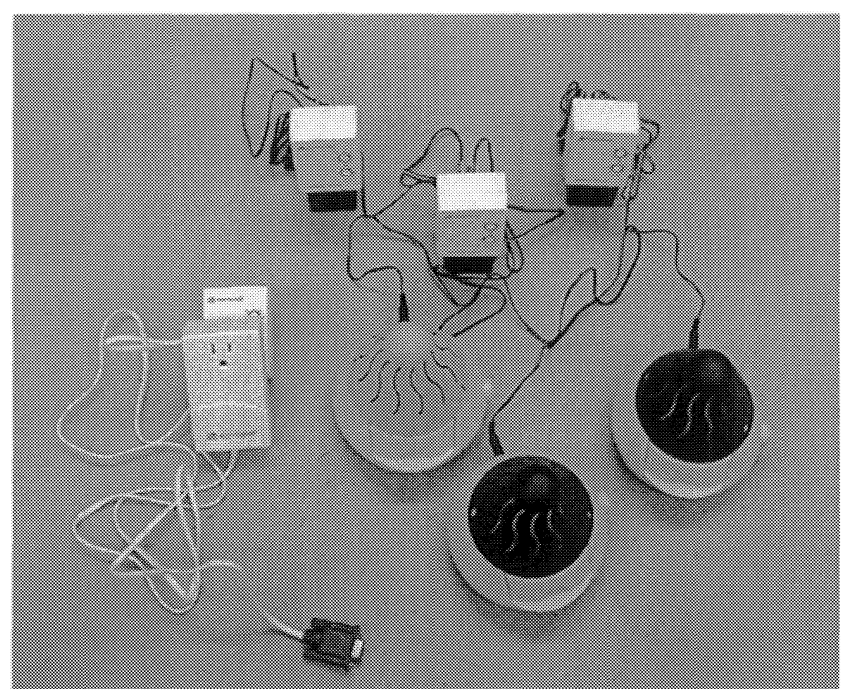

Figure 4. X-10 switching hardware shown with three aromatherapy diffusers.

More sophisticated methods were investigated but were found to be unnecessary as the gestures in yoga are very distinctive.

\subsection{Output Displays}

To generate the graphics for our visual display we used a modified screensaver called Starfish [14]. This display gave dynamic kaleidoscopic-like visuals with interesting colour patterns that we could control. The graphics were projected onto a large screen in a dim room so that users were bathed in the dominant colour. For the olfactory display, we used three aromatherapy diffusers (Spa Scenter Professional Aromatherapy Diffusers, Hubmar, Inc.). These were controlled through an X-10 interface by the GR module. The olfactory modules are shown in figure 4 . Finally, the musical output was performed using a MIDI controlled electronic keyboard synthesizer (Yamaha EX-5). We composed pieces to match the chakra theory choice of key. Likewise, we built an algorithm to ensure smooth transitions between pieces as the gestures change.

\section{Testing / User Feedback}

Tests and demonstrations of the Yoga system took place in the Human Communication Technologies Laboratory at the University of British Columbia. Four test subjects were used during testing: two were yoga practitioners and two were not. Participants reported that the gesture recognition module was pre- 
cise and reliable and that it never failed to recognize a gesture. Those with yoga experience found the kaleidoscope-like visual effects and the colour changes to be satisfactory. Colours matched with their knowledge of chakra theory. The mandalas were rated highest, however, participants also assessed the musical feedback favourably. No one found the music intrusive, and three of the subjects found that the music enhanced the experience. One of the participants was disappointed by the quality of the sound coming from the low quality speakers we used. This is easily remedied.

We were very interested in users' comments on the novel olfactory feedback. It received positive comments. However, three of the participants were unsure whether or not the scents greatly enhanced their experience. They did enjoy the scents from the diffusers but could not reliably identify which scent was displayed at any particular time. We do not take this as a negative criticism since the scents are supposed to be "in the background" and so not necessarily recognizable. Also, it is the theory of the chakras that dictates which scent to use for each posture. These may have subconscious effects that do not require users to be able to identify any particular scent. More testing and research is required for analyzing the system's olfactory feedback.

\section{Conclusions and Future Work}

Our small user group enjoyed the Yoga system and said they would like to use it again. It does appear to enhance the yoga experience, however, further development and testing is required. Our focus was on testing the integration of the various output displays with ancient, well-defined yoga practice. For future versions we will use a vision system for tracking body position so that the user will not have to contend with wires connected to a Fastrak unit. As well, we will extend the number of gestures to incorporate all seven poses. Our system does not attempt to address the validity of the therapeutic value of yoga, rather we are only exploiting the well defined theory as well as the stylized nature of the domain. Yoga is practiced by millions of people and we believe our system is an excellent step to enhance the experience though an interactive, multimedia system.

\section{References}

[1] Anandamurti. Yoga Psychology. Translated by Vijayananda Avadhuta and Jayanta Kumar. Calcutta: Ananda Marga Publications, 1990.

[2] Knapp, B., "BioMuse Neural Interface Development Platform." BioControl Systems, Inc. 400 Oyster Point Blvd., Ste. 103 South San Francisco, CA, USA 94080-1917. http://www.biocontrol.com

[3] Carlin, A.S., Hoffman, H. and Weghorst. (1997). "Virtual Reality and Tactile Augmentation in the Treatment of Spider Phobia: A Case Study." Behavior Research and Therapy, 35(2), 153-158. 
[4] Davis, J. and Bobick, A. "Virtual PAT: A Virtual Personal Aerobics Trainer." Workshop on Perceptual User Interfaces. November 1998, San Francisco, CA, USA, pp. 13-18.

[5] Fels, S. S., Reiners, D. and Mase, K. "Iamascope: An Interactive Kaleidoscope". Virtual Proceedings of SIGGRAPH97: The Electric Garden. August 1997, pp. 76-77.

[6] Hittleman, R. Richard Hittleman's Yoga: 28-Day Exercise Plan. New York: Bantam/Workman, 1969.

[7] Holistic Online. Yoga Steps and Sun Salutation. accessed on April 13, 2002. http://www.holisticonline.com/

[8] Jenkins, K. and Quinlin, D., Visual Music, Special Effects and Instructional Videos, Immediate Future Productions, CA, USA, 2001.

[9] MacLaine, S. Shirley MacLaine's Inner Workout. (Stamford, CT: High Ridge Productions / Vestron Video), 1988.

[10] Patanjali. Translated by James H. Woods. The Yoga - System of Patanjali. (Delhi: Motilal Banarsidass), 1966.

[11] Tosa, Naoko. "Unconscious Flow", ACM SIGGRAPH 99 Electronic Art and Animation Catalog, LA, CA, USA, 1999, pg 11.

[12] Selby, J. Kundalini Awakening. New York: Bantam Books, 1992.

[13] Taeger, Hans and Thomas Siegfried, Manjushri Mandala, http://www.iol.ie/ taeger/, accessed on April 13, 2002.

[14] Zawinski, Jamie, Starfish, XSceeensaver software, June 14, 1997, http://www.jwz.com accessed on April 13, 2002. 